\title{
Efficient Synthesis of O-Succinimidyl-(tert- Butoxycarbonylamino)methyl Carbamates Derived from $\alpha$-Amino Acids Accelerated by Ultrasound: Application to the Synthesis of Ureidodipeptides
}

\author{
Vommina V. Sureshbabu , Naremaddepalli S. Sudarshan \& Kantharaju
}

To cite this article: Vommina V. Sureshbabu , Naremaddepalli S. Sudarshan \& Kantharaju (2008) Efficient Synthesis of O-Succinimidyl-(tert-Butoxycarbonylamino)methyl Carbamates Derived from $\alpha$-Amino Acids Accelerated by Ultrasound: Application to the Synthesis of Ureidodipeptides, Synthetic Communications, 38:13, 2168-2184, DOI: $10.1080 / 00397910802026386$

To link to this article: http://dx.doi.org/10.1080/00397910802026386

Submit your article to this journal 주

山 Article views: 74

a

View related articles

Citing articles: 5 View citing articles $\widetilde{ }$ 


\title{
Efficient Synthesis of $\boldsymbol{O}$-Succinimidyl- (tert-Butoxycarbonylamino)methyl Carbamates Derived from $\alpha$-Amino Acids Accelerated by Ultrasound: Application to the Synthesis of Ureidodipeptides
}

\author{
Vommina V. Sureshbabu, Naremaddepalli S. Sudarshan, \\ and Kantharaju \\ Department of Studies in Chemistry, Central College Campus, \\ Bangalore University, Bangalore, India
}

\begin{abstract}
The synthesis of $O$-succinimidyl-(tert-butoxycarbonylamino)methyl carbamates employing isocyanates made through the Curtius rearrangement of Boc-amino acid azides in the presence of $N$-hydroxysuccinimide under the influence of ultrasound is described.
\end{abstract}

Keywords: Boc-amino acid azide; Boc-amino acid isocyanate; $N$-hydroxysuccinimide; Ultrasonic irradiation

\section{INTRODUCTION}

Ureidopeptides are an important class of peptidomimetics by virtue of their applications as drugs with more elevated biological properties than the parent peptides ${ }^{[1,2]}$ as well as structural motifs for conformational analysis. ${ }^{[3,4]}$ Therefore, development of new methods to synthesize these compounds has gained attention. Recently, Fishcer et al. have reported the synthesis of six $O$-succinimidyl-(tert-butoxycarbonylamino)methyl carbamates $^{[5]}$ through the Curtius rearrangement of corresponding acid azides under high-temperature reflux. In this case, the product yields have

Received August 20, 2007

Address correspondence to Vommina V. Sureshbabu, Department of Studies in Chemistry, Central College Campus, Dr B. R. Ambedkar Veedhi, Bangalore University, Bangalore 560 001, India. E-mail: hariccb@rediffmail.com 
shown to be less then satisfactory, presumably because of the decomposition of Boc-protected derivatives at the elevated temperature used for the reaction. Consequently, the preparation of isocyanates derived from Boc amino acids requires an alternative strategy. We herein demonstrate the Curtius rearrangement of Boc amino acid azides under ultrasonication at ambient temperature.

\section{RESULTS AND DISCUSSION}

Boc-/Z-amino acid azides are known for their instability even at room temperature. ${ }^{[6]}$ Their utility as peptide coupling agents at ambient temperature was accompanied by the formation of the reactive isocyanates leading to several side products. Recently, Guichard et al. reported an efficient stepwise synthesis of $N, N^{\prime}$-linked oligoureas [HN-CHR-CH$\mathrm{NH}-\mathrm{CO}]_{\mathrm{n}}$ in solution and on solid support utilizing $O$-succinimidyl carbamates derived from $N$-Boc-/Fmoc- $\beta^{3}$-amino acids as well as Boc-/Z-/Fmoc- $\alpha$-amino acids and dipeptides as activated monomers. ${ }^{[7,8]}$ Further, they have explained the short-range interaction of the cis-trans urea motif in urea peptides Boc- $L-g \operatorname{Ser}(\mathrm{Bzl})-\mathrm{CONHMe} / \mathrm{NMe}_{2}$, Piv- $L$ Pro- $L$-gLeu-CONHMe/NMe ${ }_{2}$, Boc-L-gSer(Bzl)-CO- $L$-Leu-NHMe, and Boc-L-gSer(Bzl)-CO-D-Leu-NMe ${ }_{2} \cdot{ }^{[9]}$ Subsequently, they have also described solution-phase synthesis of hexahydro-1,3,5-triazepine-2,6diones, novel rigid, highly substituted, seven-membered ring urea-based scaffolds. ${ }^{[10]}$

In the present study, the Boc- $\alpha$-amino acid 1 ( 1 equiv) was dissolved in THF $(5 \mathrm{~mL})$ and cooled to $-15^{\circ} \mathrm{C}$. After the addition of ethyl chloroformate (EtOCOCl, 1.1 equiv) and $N$-methylmorpholine (NMM, 1.1 equiv), the mixture was stirred at $-15^{\circ} \mathrm{C}$ for $20 \mathrm{~min}$. The resulting white suspension was allowed to warm to $-5^{\circ} \mathrm{C}$, treated with an aqueous solution ( $1 \mathrm{~mL}$ ) of $\mathrm{NaN}_{3}$ (2.5 equiv), and stirred for $10 \mathrm{~min}$. After usual aqueous workup, the resulting Boc- $\alpha$-amino acid azide in toluene was exposed to ultrasound at ambient temperature in the presence of an equimolar quantity of $N$-hydroxysuccinimide and NMM. Initially they rearranged to the corresponding isocyanates, which are trapped by

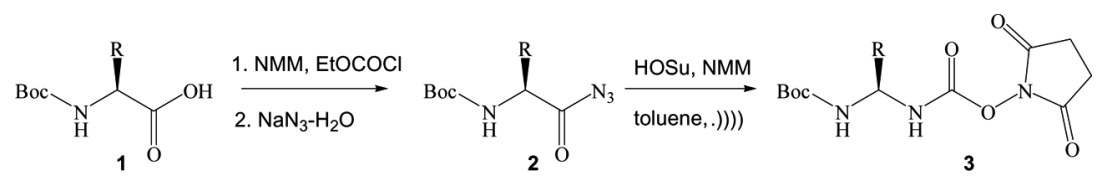

Scheme 1. Synthesis of $O$-Succinimidyl(Boc)methylcarbamates. 
$N$-hydroxysuccinimide, giving the desired $O$-succinimidyl-(tertbutoxycarbonylamino) methyl carbamate 3a-t (Scheme 1) in high yields.

The entire course of the reaction was complete in about $15-20 \mathrm{~min}$. The methyl carbamates 3 precipitated directly from the toluene solution were simply collected by filtration and washed with toluene. All the methyl carbamates 3 were characterized by IR, ${ }^{1} \mathrm{H},{ }^{13} \mathrm{C}$ NMR, and mass spectrometry. Their IR spectrum contains a characteristic sharp peak of methyl carbamate urethane stretching vibrational frequency at around

Table 1. Physical constants of O-succinimdyl-(tert-butoxycarbonylamino)methyl carbamates

\begin{tabular}{|c|c|c|c|c|c|c|}
\hline \multirow[b]{2}{*}{$\begin{array}{l}\text { Compound } \\
\mathbf{3}\end{array}$} & \multirow[b]{2}{*}{$\mathrm{R}$} & \multirow[b]{2}{*}{$\mathrm{R}^{1}$} & \multirow[b]{2}{*}{ Yield $(\%)$} & \multirow[b]{2}{*}{$\mathrm{Mp}\left({ }^{\circ} \mathrm{C}\right)$} & \multicolumn{2}{|c|}{ MALDI mass } \\
\hline & & & & & $\begin{array}{l}\text { Calculated } \\
{[\mathrm{M}]^{+}}\end{array}$ & $\begin{array}{c}\text { Found } \\
{[\mathrm{M}+\mathrm{Na}]^{+}}\end{array}$ \\
\hline $\mathrm{a}$ & $\mathrm{H}$ & $\mathrm{CH}_{3}$ & 87 & 152 & 301.3 & 324.2 \\
\hline $\mathrm{b}$ & $\mathrm{H}$ & $\mathrm{H}$ & 82 & 166 & 287.3 & 310.2 \\
\hline $\mathrm{c}$ & $\mathrm{H}$ & $\mathrm{CH}\left(\mathrm{CH}_{3}\right)_{2}$ & 85 & 107 & 329.4 & 352.3 \\
\hline $\mathrm{d}$ & $\mathrm{H}$ & $\mathrm{CH}_{2} \mathrm{CH}\left(\mathrm{CH}_{3}\right)_{2}$ & 84 & 125 & 343.4 & 366.3 \\
\hline $\mathrm{e}$ & $\mathrm{H}$ & $\mathrm{CH}\left(\mathrm{CH}_{3}\right) \mathrm{C}_{2} \mathrm{H}_{5}$ & 82 & 129 & 343.4 & 366.2 \\
\hline $\mathrm{f}$ & $\mathrm{H}$ & $\mathrm{CH}_{2} \mathrm{C}_{6} \mathrm{H}_{5}$ & 86 & 143 & 377.4 & 400.3 \\
\hline $\mathrm{g}$ & $\mathrm{H}$ & $\mathrm{C}_{6} \mathrm{H}_{5}$ & 87 & 126 & 363.4 & 386.3 \\
\hline $\mathrm{h}^{*}$ & $\mathrm{H}$ & $\mathrm{C}_{6} \mathrm{H}_{5}$ & 85 & 122 & 363.4 & 386.3 \\
\hline $\mathrm{i}$ & $\mathrm{H}$ & $\mathrm{CH}_{2} \mathrm{OBzl}$ & 83 & 141 & 407.4 & 430.2 \\
\hline $\mathrm{j}$ & $\mathrm{H}$ & $\mathrm{CH}_{2} \mathrm{COOBzl}$ & 84 & 122 & 435.4 & 458.5 \\
\hline $\mathrm{k}$ & $\mathrm{H}$ & $\left(\mathrm{CH}_{2}\right)_{2} \mathrm{COOBzl}$ & 80 & 140 & 449.5 & 472.2 \\
\hline 1 & $\mathrm{R}=\mathrm{R}^{1}$ & $-\left(\mathrm{CH}_{2}\right)_{3}=$ & 82 & 127 & 327.3 & 350.3 \\
\hline $\mathrm{m}$ & $\mathrm{H}$ & $\left(\mathrm{CH}_{2}\right)_{2} \mathrm{SCH}_{3}$ & 80 & 125 & 347.4 & 370.3 \\
\hline $\mathrm{n}$ & $\mathrm{H}$ & $\left(\mathrm{CH}_{2}\right)_{4} \mathrm{NHZ}$ & 75 & 149 & 492.5 & 515.2 \\
\hline $\mathrm{o}$ & $\mathrm{H}$ & $\mathrm{CH}_{2} \mathrm{C}_{6} \mathrm{H}_{5} \mathrm{OH}$ & 72 & 151 & 393.4 & 416.3 \\
\hline $\mathrm{p}$ & $\mathrm{H}$ & $\mathrm{CH}\left(\mathrm{CH}_{3}\right) \mathrm{OH}$ & 76 & 142 & 331.3 & 354.3 \\
\hline q & $\mathrm{H}$ & 1) & 70 & 168 & 550.5 & 573.2 \\
\hline $\mathrm{r}$ & $\mathrm{H}$ & $\left(\mathrm{CH}_{2}\right)_{2} \mathrm{CONH}_{2}$ & 72 & 136 & 358.3 & 381.3 \\
\hline $\mathrm{s}$ & $\mathrm{H}$ & $\mathrm{CH}_{2} \mathrm{CONH}_{2}$ & 75 & 141 & 344.3 & 367.1 \\
\hline $\mathrm{t}$ & $\mathrm{H}$ & & 70 & 149 & 501.4 & 524.3 \\
\hline
\end{tabular}

Notes: Phg $=$ phenylglycine; ${ }^{*} \mathrm{D}$-configuration. 
Table 2. Comparison of Boc-gPhe-COOSu (3f) formation under different methods

\begin{tabular}{lcc}
\hline Methods & Time $(\min )$ & Yield $(\%)$ \\
\hline Thermal & 45 & 43 \\
Microwave & 2 & 49 \\
Ultrasound & 25 & 86 \\
\hline
\end{tabular}

$1735-1745 \mathrm{~cm}^{-1}$. The physical constants as well as yields $70-85 \%$ of methyl carbamates are given in the Table 1. Almost all the proteniogenic amino acids including Ser, Thr, Tyr, Asp, Glu, Asn, Gln, His, Trp, and Cys have been prepared. The methyl carbamates 3 were stable white crystalline solids. When stored at $\mathrm{rt}$ for long periods, neither noticeable degradation nor any change in their spectral behavior was observed. A comparative study for the synthesis of $O$-succinimidyl-\{1-(tertbutoxycarbonylamino)-2-phenyl\} methylcarbamate using Boc-Phe- $\mathrm{N}_{3}$ and N-hydroxysuccinimide was carried out under different conditions. It was found that the formation of $\mathbf{3}$ through Curtius rearrangement assisted by ultrasound under ambient temperature results in better yields then the classical thermal method or microwave irradiation, and results are furnished in Table 2.

To demonstrate the use of the activated carbamates, the synthesis of Boc-dipeptidyl urea esters has been carried out. The reaction of methylcarbamate 3 with amino acid esters $\mathbf{4}$ in THF-DMF (2:1) in the presence of NMM at rt gave the corresponding ureas $\mathbf{5}$ in good yields (Scheme 2). The reaction proceeds rapidly and was found to be completed within 30 min at rt. A routine workup and recrystallization with DMSO-water resulted in analytically pure dipeptidyl urea esters $\mathbf{5 a}-\mathbf{j}$. The only byproduct formed during the reaction was $N$-hydroxysuccinimide, which was easily removed during aqueous wash. All the dipeptidyl ureas prepared are characterized by ${ }^{1} \mathrm{H}$ and ${ }^{13} \mathrm{C}$ NMR and mass spectral methods.

The carbamates were further reacted with the in situ generated $N$, $O$-bis(trimethylsilyl)amino acids 7 to synthesize Boc-dipeptidyl urea acids

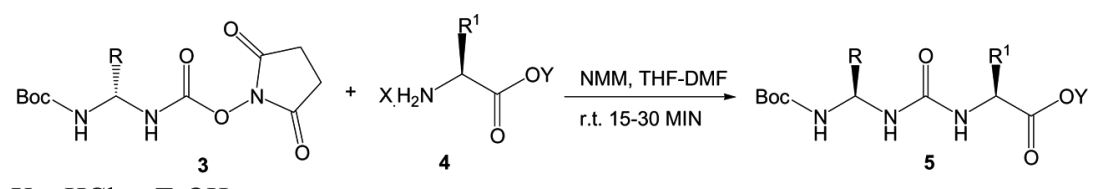
$\mathrm{X}=\mathrm{HCl}, p-\mathrm{TsOH}$

Scheme 2. Synthesis of ureidopeptide esters from 3. 
Table 3. List of ureidopeptide esters prepared through scheme 2

\begin{tabular}{llll}
\hline Urea ester 5 & \multicolumn{1}{c}{$\mathrm{R}$} & \multicolumn{1}{c}{$\mathrm{R}^{1}$} & $\mathrm{Y}$ \\
\hline $\mathbf{a}$ & $\mathrm{CH}(\mathrm{CH} 3) 2$ & $\mathrm{CH} 3$ & $\mathrm{CH} 3$ \\
$\mathbf{b}$ & $\mathrm{CH} 2 \mathrm{C} 6 \mathrm{H} 5$ & $\mathrm{CH}(\mathrm{CH} 3) 2$ & $\mathrm{CH} 3$ \\
$\mathbf{c}$ & $\mathrm{H}$ & $\mathrm{CH} 2 \mathrm{C} 6 \mathrm{H} 5$ & $\mathrm{CH} 3$ \\
$\mathbf{d}$ & $\mathrm{CH} 2 \mathrm{C} 6 \mathrm{H} 5$ & $\mathrm{CH} 3$ & $\mathrm{CH} 3$ \\
$\mathbf{e}$ & $\mathrm{CH} 2 \mathrm{OBzl}$ & $\mathrm{H}$ & $\mathrm{CH} 3$ \\
$\mathbf{f}$ & $\mathrm{CH} 2 \mathrm{COOBzl}$ & $\mathrm{H}$ & $\mathrm{CH} 3$ \\
$\mathbf{g}$ & $(\mathrm{CH} 2) 2 \mathrm{COOBzl}$ & $\mathrm{CH} 2 \mathrm{CH}(\mathrm{CH} 3) 2$ & $\mathrm{CH} 3$ \\
$\mathbf{h}$ & $\mathrm{CH}(\mathrm{CH}) \mathrm{C} 2 \mathrm{H} 5$ & $\mathrm{CH} 2 \mathrm{C} 6 \mathrm{H} 5$ & $\mathrm{CH} 3$ \\
$\mathbf{i}$ & $\mathrm{CH} 2 \mathrm{C} 6 \mathrm{H} 4 \mathrm{OH}$ & $\mathrm{CH} 3$ & $\mathrm{CH} 3$ \\
& & & $\mathrm{CH} 3$ \\
$\mathbf{j}$ & $\mathrm{H}_{2} \mathrm{C}$ & $\mathrm{CH}(\mathrm{CH} 3) 2$ & \\
\hline
\end{tabular}

(Scheme 3). A simple workup of the reaction mixture followed by their recrystallization resulted in pure dipeptidyl urea acids $\mathbf{8 a - d}$, which were fully characterized.

\section{Racemization Study for Methylcarbamates 3}

To demonstrate the stereospecificity of both the carbamates $\mathbf{3}$ prepared and their use as urea coupling agents, the carbamate $\mathbf{3 f}$ derived from Boc-Phe was coupled with R- $(+)$, S- $(-)$, and R,S- $( \pm)-1$-phenylethylamines $9 \mathbf{a}-\mathbf{c}$ to obtain the white solid ureas $10 \mathrm{a}, \mathbf{1 0 b}$, and $10 \mathbf{c}$ respectively (Scheme 4, Figure 1), and their recrystallization resulted in analytically

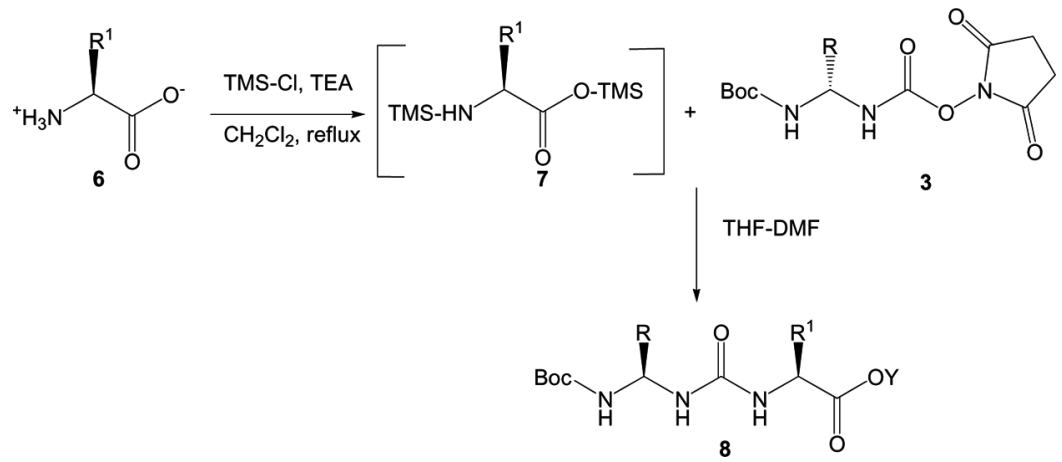

Scheme 3. Synthesis of ureidopeptide acids $8(\mathrm{Y}-\mathrm{H})$ from 3. 
Table 4. List of ureidopeptide acids obtained via scheme 3

\begin{tabular}{lll}
\hline Urea acid 8 & \multicolumn{1}{c}{$\mathrm{R}$} & \multicolumn{1}{c}{$\mathrm{R}^{1}$} \\
\hline a & $\mathrm{CH}_{2} \mathrm{CH}\left(\mathrm{CH}_{3}\right)_{2}$ & $\mathrm{CH}_{3}$ \\
b & $\mathrm{CH}_{2} \mathrm{C}_{6} \mathrm{H}_{5}$ & $\mathrm{CH}_{3}$ \\
c & $\mathrm{CH}_{2} \mathrm{CH}\left(\mathrm{CH}_{3}\right)_{2}$ & $\mathrm{H}$ \\
d & $\mathrm{CH}\left(\mathrm{CH}_{3}\right)_{2}$ & $\mathrm{CH}_{2} \mathrm{C}_{6} \mathrm{H}_{5}$ \\
\hline
\end{tabular}

pure compounds. Their ${ }^{1} \mathrm{H}$ NMR analysis revealed that the methyl group resonances of $\mathbf{1 0 a}(\delta, 1.28$ and 1.30$)$ and $\mathbf{1 0 b}(\delta, 1.26$ and 1.28$)$ are clearly separated by $0.02 \mathrm{ppm}$ in DMSO- $\mathrm{d}_{6}$ solution. In addition, the ${ }^{1} \mathrm{H}$ NMR spectrum of the racemic urea 10c was observed as a doublet of doublet for the methyl group resonances at $(\delta, 1.25,1.27,1.28$, and 1.30$)$. This clearly confirms that both the carbamates' preparation as well as the coupling reaction to obtain the peptidyl ureas are completely free from racemization.

\section{EXPERIMENTAL}

The melting points were recorded in open capillary tubes and are uncorrected. The reactions were carried out using a sonic bath $(35 \mathrm{kHz}$, Elma, $\mathrm{T} 310 / \mathrm{H}$ German make) at ambient temperature. Infrared spectra were recorded on a Nicolet Impact 400D FT-IR spectrometer (KBr pellets, $3 \mathrm{~cm}^{-1}$ resolution). Elementalanalyses were carried out using a PerkinElmer analyzer, and the samples were dried for $24 \mathrm{~h}$ under vacuum before analysis. ${ }^{1} \mathrm{H}$ and ${ }^{13} \mathrm{C}$ NMR spectra were recorded on a Bruker AMX 400$\mathrm{MHz}$ spectrometer. Mass spectra were recorded on Maldi, PE-Sciex 150 EX LC-MS, and Jeol-JMS-600H spectrometers in $\mathrm{FAB}^{+}$mode using 3-nitrobenzyl alcohol as a liquid matrix. All solvents were freshly distilled prior to use. Amino acid methyl ester hydrochlorides were prepared using methanol and thionyl chloride.<smiles>CC(C)(C)OC(=O)N[C@@H](Cc1ccccc1)NC(=O)ON1C(=O)CCC1=O</smiles><smiles>CC(N)c1ccccc1</smiles><smiles>CC(NC(=O)N[C@@H](Cc1ccccc1)NC(C)(C)C)c1ccccc1</smiles>

Scheme 4. Preparation of urea adducts for racemization studies. 


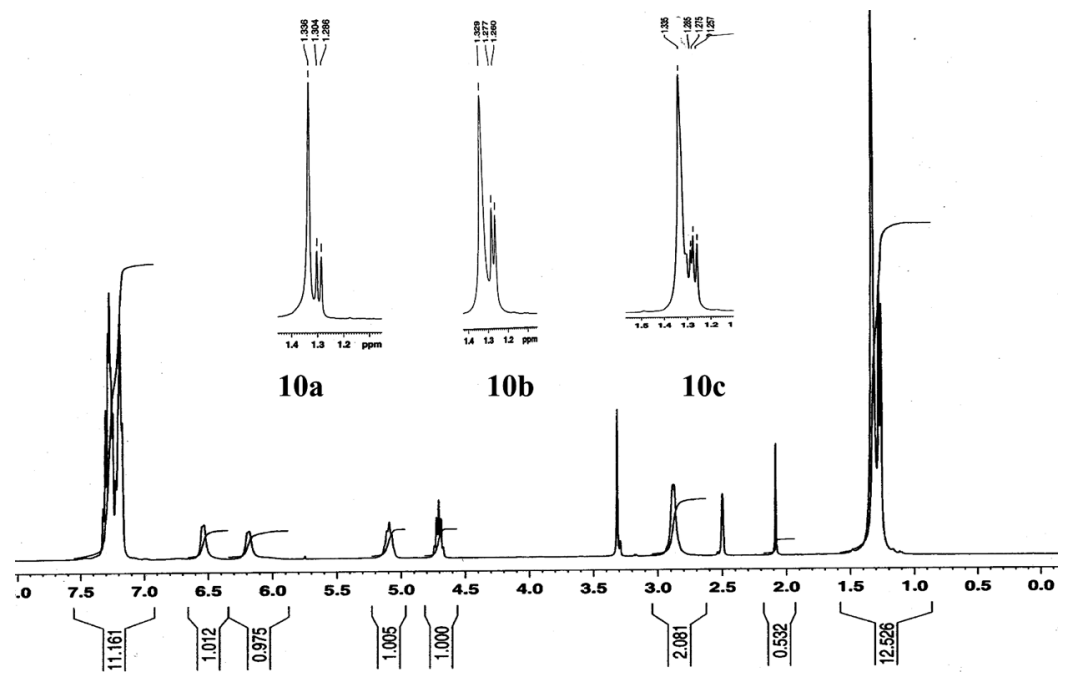

Figure 1. ${ }^{1} \mathrm{H}$ NMR spectra of Boc-Phe- $\psi(\mathrm{NH}-\mathrm{CO}-\mathrm{NH})-\mathrm{R}-(+)-1-$ phenylethylamine: Inset figure shown splitting pattern of $\mathrm{CH} 3$ group of 1-phenylethylamine in urea adducts $\mathrm{R}-(+)-1$-phenylethylamine (10a), S- $(-)-1$-phenylethylamine (10b), and R,S- $( \pm$ )-1-phenylethylamine (10c).

\section{General Procedure for the Synthesis of $\boldsymbol{O}$-Succinimidyl- (tert-butoxycarbonylamino)methylcarbamates 3a-t}

Boc- $\alpha$-amino acid 1 ( $1 \mathrm{mmol})$ was dissolved in THF $(5 \mathrm{~mL})$ and cooled to $-20{ }^{\circ} \mathrm{C}$. After addition of ethylchloroformate $(1.1 \mathrm{mmol})$ and $N$ methylmorpholine $(1.1 \mathrm{mmol})$, the mixture was stirred at $-20{ }^{\circ} \mathrm{C}$ for $15 \mathrm{~min}$ and allowed to warm to $-5^{\circ} \mathrm{C}$. It was treated with an aqueous solution of $\mathrm{NaN}_{3}(2.5 \mathrm{mmol}$ in $2 \mathrm{~mL}$ of water) for $5 \mathrm{~min}$. After concentration of the solvent under reduced pressure, the resulting residue was diluted with $\mathrm{CH}_{2} \mathrm{Cl}_{2}(15 \mathrm{~mL})$; washed with $5 \%$ citric acid solution $(3 \times 5 \mathrm{~mL}), \quad 5 \%$ sodium bicarbonate solution $(3 \times 5 \mathrm{~mL})$, and brine; dried over anhydrous $\mathrm{Na}_{2} \mathrm{SO}_{4}$; and concentrated under reduced pressure to give the acyl azide $\mathbf{2}$, which was used without further purification. The acyl azide was taken in toluene $(5 \mathrm{~mL})$, and $N$-hydroxy succinimide ( $1 \mathrm{mmol})$ and NMM (1.1 mmol) were successively added. The mixture was sonicated in an ultrasound bath for about 15-25 min at ambient temperature. After the completion of the reaction, carbamates crystallized from the toluene solution were collected by filtration. All the carbamates 3 have been fully characterized. The physical and spectral data are furnished in Table 1. 


\section{Data}

\section{Boc-gAla-COOSu (3a)}

White solid,IR: $1715,1745,1780 \mathrm{~cm}^{-1} ;{ }^{1} \mathrm{H}$ NMR (DMSO): $\delta 1.16(3 \mathrm{H}$, s), $1.36(9 \mathrm{H}, \mathrm{s}), 2.8(4 \mathrm{H}, \mathrm{s}), 3.8(1 \mathrm{H}, \mathrm{m}), 5.2(1 \mathrm{H}, \mathrm{m}), 8.5(1 \mathrm{H}, \mathrm{d}) ;{ }^{13} \mathrm{C}$ NMR (DMSO): $\delta 18.3,28.6,59.6,70.1,72.2,78.4,151.2,154.7,170.8$. Anal. calcd. for $\mathrm{C}_{12} \mathrm{H}_{19} \mathrm{~N}_{3} \mathrm{O}_{6}: \mathrm{C}, 47.84 ; \mathrm{H}, 6.36 ; \mathrm{N}, 13.95$. Found: $\mathrm{C}$, 47.12; H, 6.62; N, $13.24 \%$.

\section{Boc-gGly-COOSu (3b)}

White solid,IR: $1710,1750,1775 \mathrm{~cm}^{-1} ;{ }^{1} \mathrm{H}$ NMR (DMSO): $\delta 1.36(9 \mathrm{H}$, s), $2.5(2 \mathrm{H}, \mathrm{m}), 2.8(4 \mathrm{H}, \mathrm{s}), 5.2(1 \mathrm{H}, \mathrm{br}), 8.2(1 \mathrm{H}, \mathrm{br}) ;{ }^{13} \mathrm{C}$ NMR (DMSO): $\delta$ 27.8, 40.4, 69.6, 72.0, 79.1, 151.2, 155.7, 170.5. Anal. calcd. for $\mathrm{C}_{11} \mathrm{H}_{17} \mathrm{~N}_{3} \mathrm{O}_{6}: \mathrm{C}, 45.99 ; \mathrm{H}, 5.96 ; \mathrm{N}, 14.63$. Found: $\mathrm{C}, 45.12 ; \mathrm{H}, 5.62 ; \mathrm{N}$, $14.50 \%$.

\section{Boc-gVal-COOSu (3c)}

White solid,IR: $1705,1745,1770 \mathrm{~cm}^{-1} ;{ }^{1} \mathrm{H}$ NMR (DMSO): $\delta 0.93(6 \mathrm{H}, \mathrm{t})$, $1.35(9 \mathrm{H}, \mathrm{s}), 1.9(1 \mathrm{H}, \mathrm{m}), 2.7(4 \mathrm{H}, \mathrm{s}), 3.9(1 \mathrm{H}, \mathrm{m}), 5.3(1 \mathrm{H}, \mathrm{br}), 8.4(1 \mathrm{H}$, d); ${ }^{13} \mathrm{C}$ NMR (DMSO): $\delta 18.5,19.7,28.4,29.6,58.6,69.7,72.1,79.0$, 150.7, 155.2, 170.8. Anal. Calcd. for $\mathrm{C}_{14} \mathrm{H}_{23} \mathrm{~N}_{3} \mathrm{O}_{6}: \mathrm{C}, 51.06 ; \mathrm{H}, 7.04 ; \mathrm{N}$, 12.76. Found: C, 51.12; H, 7.62; N, 12.24\%.

\section{Boc-gLeu-COOSu (3d)}

White solid,IR: $1715,1755,1780 \mathrm{~cm}^{-1} ;{ }^{1} \mathrm{H}$ NMR (DMSO): $\delta 0.93(6 \mathrm{H}$, d), $1.25-1.35(11 \mathrm{H}, \mathrm{m}), 1.62(1 \mathrm{H}, \mathrm{m}), 2.7(4 \mathrm{H}, \mathrm{s}), 3.7(1 \mathrm{H}, \mathrm{m}), 5.2(1 \mathrm{H}$, br), $8.5(1 \mathrm{H}, \mathrm{br}) ;{ }^{13} \mathrm{C}$ NMR (DMSO): $\delta 22.1,23.4,24.8,41.0,51.6$, 70.1, 72.2, 78.7, 151.2, 154.7, 171.0. Anal. calcd. for $\mathrm{C}_{15} \mathrm{H}_{25} \mathrm{~N}_{3} \mathrm{O}_{6}: \mathrm{C}$, 52.47; H, 7.34; N, 12.24. Found: C, 52.12; H, 7.62; N, 12.50\%.

\section{Boc-gIle-COOSu (3e)}

White solid,IR: $1715,1755,1780 \mathrm{~cm}^{-1} ;{ }^{1} \mathrm{H}$ NMR (DMSO): $\delta 0.91(6 \mathrm{H}$, $\mathrm{m}), 1.12(1 \mathrm{H}, \mathrm{m}), 1.3(9 \mathrm{H}, \mathrm{s}), 1.52(2 \mathrm{H}, \mathrm{m}), 3.7(1 \mathrm{H}, \mathrm{m}), 4.94(1 \mathrm{H}, \mathrm{m})$, $5.2(1 \mathrm{H}, \mathrm{br}) ;{ }^{13} \mathrm{C}$ NMR (DMSO): $\delta 11.5,15.7,25.2,26.2,28.6,34.4$, 56.6, 78.2, 151.2, 153.7, 170.5. Anal. calcd. for $\mathrm{C}_{15} \mathrm{H}_{25} \mathrm{~N}_{3} \mathrm{O}_{6}$ : C, 52.47; $\mathrm{H}, 7.34$; N, 12.24. Found: C, 53.12; H, 7.0; N, $12.10 \%$. 
Boc-gPhe-COOSu (3f)

White solid, IR: $1715,1755,1780 \mathrm{~cm}^{-1} ;{ }^{1} \mathrm{H}$ NMR (DMSO): $\delta 1.36(9 \mathrm{H}$, m), $2.7(4 \mathrm{H}, \mathrm{s}), 2.8(2 \mathrm{H}, \mathrm{d}), 3.9(1 \mathrm{H}, \mathrm{m}), 5.0(1 \mathrm{H}, \mathrm{d}), 5.4(1 \mathrm{H}, \mathrm{m}), 7.25-7.3$ $(5 \mathrm{H}, \mathrm{m}) ;{ }^{13} \mathrm{C}$ NMR (DMSO): $\delta 26.1,28.9,62.2,79.1,126.9,127.6,128.9$, $129.0,129.1,130.2,137.9,151.4,155.2,171.6$. Anal. calcd. for $\mathrm{C}_{18} \mathrm{H}_{23} \mathrm{~N}_{3} \mathrm{O}_{6}: \mathrm{C}, 57.29 ; \mathrm{H}, 6.14 ; \mathrm{N}, 11.13$. Found: $\mathrm{C}, 57.12 ; \mathrm{H}, 6.20 ; \mathrm{N}$, $11.24 \%$.

\section{Boc-gPhg-COOSu (3g)}

White solid, IR: $1715,1755,1780 \mathrm{~cm}^{-1} ;{ }^{1} \mathrm{H}$ NMR (DMSO): $\delta 1.3(9 \mathrm{H}, \mathrm{s})$, $2.68(4 \mathrm{H}, \mathrm{s}), 3.9(1 \mathrm{H}, \mathrm{m}), 4.9(1 \mathrm{H}, \mathrm{d}), 5.2(1 \mathrm{H}, \mathrm{br}), 7.25-7.3(5 \mathrm{H}, \mathrm{m}) ;{ }^{13} \mathrm{C}$ NMR (DMSO): $\delta$ 26.6, 28.6, 57.8, 78.2, 125.8, 127.1, 127.9, 129.7, 151.2, 154.7, 171.5. Anal. calcd. for $\mathrm{C}_{17} \mathrm{H}_{21} \mathrm{~N}_{3} \mathrm{O}_{6}$ : C, 56.19; H, 5.83; N, 11.56 . Found: C, 56.12; H, 5.62; N, 11.24\%.

\section{Boc-g(D)Phg-COOSu (3h*)}

White solid, IR: $1715,1755,1780 \mathrm{~cm}^{-1} ;{ }^{1} \mathrm{H}$ NMR (DMSO): $\delta 1.28(9 \mathrm{H}$, m), $2.7(4 \mathrm{H}, \mathrm{s}), 3.88(1 \mathrm{H}, \mathrm{m}), 4.8(1 \mathrm{H}, \mathrm{m}), 5.1(1 \mathrm{H}, \mathrm{br}), 7.25-7.3(5 \mathrm{H}, \mathrm{m})$;

${ }^{13} \mathrm{C}$ NMR (DMSO): $\delta 26.7,28.8,58.6,79.0,125.6,127.1,127.9,129.7$, 151.8, 155.7, 170.9. Anal. calcd. for $\mathrm{C}_{17} \mathrm{H}_{21} \mathrm{~N}_{3} \mathrm{O}_{6}: \mathrm{C}, 56.19 ; \mathrm{H}, 5.83 ; \mathrm{N}$, 11.56. Found: C, 56.10; H, 5.66; N, $11.20 \%$.

\section{Boc-gSer(Bzl)-COOSu (3i)}

White solid, IR: $1715,1755,1785 \mathrm{~cm}^{-1} ;{ }^{1} \mathrm{H}$ NMR (DMSO): $\delta 1.3(9 \mathrm{H}, \mathrm{s})$, $3.6(2 \mathrm{H}, \mathrm{d}), 3.8(1 \mathrm{H}, \mathrm{m}), 4.8(2 \mathrm{H}, \mathrm{s}), 5.1(1 \mathrm{H}, \mathrm{d}), 5.3(1 \mathrm{H}, \mathrm{br}), 7.25-7.30$ $(5 \mathrm{H}, \mathrm{m}) ;{ }^{13} \mathrm{C}$ NMR (DMSO): $\delta 25.6,27.8,51.4,62.6,78.2,124.5,125.1$, 127.1, 127.9, 151.2, 153.9, 171.5. Anal. calcd. for $\mathrm{C}_{19} \mathrm{H}_{25} \mathrm{~N}_{3} \mathrm{O}_{7}$ : C, 56.01; H, 6.18; N, 10.31. Found: C, 56.10; H, 6.62; N, 10.70\%.

\section{Boc-gAsp(Bzl)-COOSu (3j)}

White solid, IR: $1715,1755,1780 \mathrm{~cm}^{-1} ;{ }^{1} \mathrm{H}$ NMR (DMSO): $\delta 1.3(9 \mathrm{H}, \mathrm{s})$, $3.6(2 \mathrm{H}, \mathrm{d}), 3.8(1 \mathrm{H}, \mathrm{m}), 4.0(2 \mathrm{H}, \mathrm{s}), 4.9(1 \mathrm{H}, \mathrm{d}), 5.1(1 \mathrm{H}, \mathrm{m}), 7.25-7.35$ $(5 \mathrm{H}, \mathrm{m}) ;{ }^{13} \mathrm{C}$ NMR (DMSO): $\delta 26.0,28.6,37.2,48.4,57.6,78.6,125.1$, 127.1, 127.9, 129.7, 151.2, 153.7, 154.8, 170.5. Anal. calcd. for $\mathrm{C}_{20} \mathrm{H}_{25} \mathrm{~N}_{3} \mathrm{O}_{8}: \mathrm{C}, 59.19 ; \mathrm{H}, 4.59 ; \mathrm{N}, 5.11$. Found: $\mathrm{C}, 59.12 ; \mathrm{H}, 4.62 ; \mathrm{N}$, $5.24 \%$. 


\section{Boc-gGlu(Bzl)-COOSu (3k)}

White solid, IR: $1715,1755,1785 \mathrm{~cm}^{-1} ;{ }^{1} \mathrm{H}$ NMR (DMSO): $\delta 1.32(9 \mathrm{H}$, s), 3.6-3.8 $(5 \mathrm{H}, \mathrm{m}), 4.9(2 \mathrm{H}, \mathrm{s}), 5.1(1 \mathrm{H}, \mathrm{d}), 5.3(1 \mathrm{H}, \mathrm{m}), 7.25-7.35(5 \mathrm{H}$, $\mathrm{m}) ;{ }^{13} \mathrm{C}$ NMR (DMSO): $\delta$ 25.8, 28.2, 35.6, 37.5, 48.8, 56.9, 78.6, 125.1, 127.1, 127.9, 129.7, 151.2, 153.7, 154.8, 171.5. Anal. calcd. for $\mathrm{C}_{21} \mathrm{H}_{27} \mathrm{~N}_{3} \mathrm{O}_{8}: \mathrm{C}, 56.12 ; \mathrm{H}, 6.06 ; \mathrm{N}, 9.35$. Found: $\mathrm{C}, 56.00 ; \mathrm{H}, 6.62 ; \mathrm{N}$, $9.24 \%$.

\section{Boc-gPro-COOSu (3l)}

White solid, IR: $1710,1745,1780 \mathrm{~cm}^{-1} ;{ }^{1} \mathrm{H}$ NMR (DMSO): $\delta 1.2-2.2$ $(13 \mathrm{H}, \mathrm{m}), 2.7(4 \mathrm{H}, \mathrm{s}), 3.4-3.7(3 \mathrm{H}, \mathrm{m}), 4.8(1 \mathrm{H}, \mathrm{d}), 5.1(1 \mathrm{H}, \mathrm{m}) ;{ }^{13} \mathrm{C}$ NMR (DMSO): $\delta 24.2,25.8,28.2,28.4,60.6,78.1,150.8,154.7,170.4$. Anal. calcd. for $\mathrm{C}_{14} \mathrm{H}_{21} \mathrm{~N}_{3} \mathrm{O}_{6}$ : C, 51.37; H, 6.47; N, 12.84. Found: $\mathrm{C}$, $51.12 ; \mathrm{H}, 6.62 ; \mathrm{N}, 12.24 \%$.

\section{Boc-gMet-COOSu (3m)}

White solid, IR: $1710,1750,1785 \mathrm{~cm}^{-1} ;{ }^{1} \mathrm{H}$ NMR (DMSO): $\delta 1.31-2.25$ $(11 \mathrm{H}, \mathrm{m}), 2.7(4 \mathrm{H}, \mathrm{s}), 3.3-3.5(4 \mathrm{H}, \mathrm{m}), 4.9(1 \mathrm{H}, \mathrm{d}), 5.15(1 \mathrm{H}, \mathrm{m}) ;{ }^{13} \mathrm{C}$ NMR (DMSO): $\delta$ 26.1, 27.9, 28.5, 48.5, 49.1, 59.8, 73.9, 151.8, 154.7, 171.4. Anal. calcd. for $\mathrm{C}_{13} \mathrm{H}_{21} \mathrm{~N}_{3} \mathrm{O}_{6} \mathrm{~S}: \mathrm{C}, 44.95 ; \mathrm{H}, 6.09 ; \mathrm{N}, 12.09$. Found: C, 45.12; H, 6.02; N, 12.06\%.

\section{Boc-gLys(Z)-COOSu (3n)}

White solid, IR: $1715,1750,1785 \mathrm{~cm}^{-1} ;{ }^{1} \mathrm{H}$ NMR (DMSO): $\delta 1.37(9 \mathrm{H}$, s), $1.6(6 \mathrm{H}, \mathrm{m}), 3.0(6 \mathrm{H}, \mathrm{m}), 4.8(2 \mathrm{H}, \mathrm{s}), 5.0(1 \mathrm{H}, \mathrm{s}), 5.4(1 \mathrm{H}, \mathrm{m}), 5.8(1 \mathrm{H}$, $\mathrm{m}), 7.25-7.30(5 \mathrm{H}, \mathrm{m}) ;{ }^{13} \mathrm{C}$ NMR (DMSO): $\delta 18.9,24.1,25.3,27.8,32.1$, 47.4, 59.7, 69.0, 79.4, 127.7, 128.4, 137.4, 152.4, 156.6, 158.3, 172.9. Anal. calcd. for $\mathrm{C}_{23} \mathrm{H}_{32} \mathrm{~N}_{4} \mathrm{O}_{8}$ : C, 56.09; $\mathrm{H}, 6.55 ; \mathrm{N}, 11.38 ; \mathrm{O}, 25.99$. Found: $\mathrm{C}$, $56.01 ; \mathrm{H}, 6.52 ; \mathrm{N}, 11.30 ; \mathrm{O}, 25.91 \%$.

\section{Boc-gTyr-COOSu (3o)}

White solid, IR: $1715,1745,1785 \mathrm{~cm}^{-1} ;{ }^{1} \mathrm{H}$ NMR (DMSO): $\delta 1.37(9 \mathrm{H}$, s), $3.0(4 \mathrm{H}, \mathrm{m}), 7.1(4 \mathrm{H}, \mathrm{m}), 5.8(1 \mathrm{H}, \mathrm{m}) ;{ }^{13} \mathrm{C}$ NMR (DMSO): $\delta 26.1,28.1$, 40.1, 63.9, 81.1, 116.0, 129.3, 129.4, 152.3, 154.8, 158.2, 172.2. Anal. calcd. for $\mathrm{C}_{18} \mathrm{H}_{23} \mathrm{~N}_{3} \mathrm{O}_{7}: \mathrm{C}, 54.96 ; \mathrm{H}, 5.89 ; \mathrm{N}, 10.68 ; \mathrm{O}, 28.47$. Found: C, 54.90; H, 5.81; N, 10.70; O, 28.51\%. 


\section{Boc-gThr-COOSu (3p)}

White solid, IR: $1710,1745,1785 \mathrm{~cm}^{-1} ;{ }^{1} \mathrm{H}$ NMR (DMSO): $\delta 1.2(3 \mathrm{H}, \mathrm{s})$, $1.38(9 \mathrm{H}, \mathrm{s}), 3.0(4 \mathrm{H}, \mathrm{m}), 3.7(1 \mathrm{H}, \mathrm{m}), 5.2(1 \mathrm{H}, \mathrm{m}), 5.8(1 \mathrm{H}, \mathrm{m}) ;{ }^{13} \mathrm{C} \mathrm{NMR}$ (DMSO): $\delta 17.9,26.1,28.2,68.6,70.8,81.1,153.1,158.2,172.8$. Anal. calcd. for $\mathrm{C}_{13} \mathrm{H}_{21} \mathrm{~N}_{3} \mathrm{O}_{7}: \mathrm{C}, 47.13 ; \mathrm{H}, 6.39 ; \mathrm{N}, 12.68 ; \mathrm{O}, 33.80$. Found: C, 47.08; H, 6.42; N, 12.70; O, 33.73\%.

\section{Boc-gTrp(Z)-COOSu (3q)}

White solid, IR: $1719,1745,1785 \mathrm{~cm}^{-1} ;{ }^{1} \mathrm{H}$ NMR (DMSO): $\delta 1.37(9 \mathrm{H}$, s), $3.0(4 \mathrm{H}, \mathrm{m}), 3.4(2 \mathrm{H}, \mathrm{m}), 5.6(\mathrm{~m}, \mathrm{H}), 7.0-7.9(10 \mathrm{H}, \mathrm{m}) ;{ }^{13} \mathrm{C} \mathrm{NMR}$ (DMSO): $\delta 25.5,28.1,33.5,63.4,64.6,81.0,108.9,119.2,122.2,122.5$, 124.8, 127.6, 128.5, 127.9, 137.7, 146.8, 148.1, 153.8, 159.3, 172.2. Anal. calcd. for $\mathrm{C}_{28} \mathrm{H}_{30} \mathrm{~N}_{4} \mathrm{O}_{8}: \mathrm{C}, 61.08 ; \mathrm{H}, 5.49 ; \mathrm{N}, 10.18 ; \mathrm{O}, 23.25$. Found: C, 61.01; H, 5.50; N, 10.10; O, 23.33\%.

\section{Boc-gGln-COOSu (3r)}

White solid, IR: $1710,1750,1785 \mathrm{~cm}^{-1} ;{ }^{1} \mathrm{H}$ NMR (DMSO): $\delta 1.36(9 \mathrm{H}$, s), $2.1(2 \mathrm{H}, \mathrm{m}), 2.4(2 \mathrm{H}, \mathrm{t}), 3.1(4 \mathrm{H}, \mathrm{m}), 5.0(2 \mathrm{H}, \mathrm{s}), 5.8(\mathrm{~m}, 1 \mathrm{H}) ;{ }^{13} \mathrm{C}$ NMR (DMSO): $\delta 16.1,25.5,28.1,32.6,62.4,81.0,152.4,158.6,172.2$, 172.4. Anal. calcd. for $\mathrm{C}_{14} \mathrm{H}_{22} \mathrm{~N}_{4} \mathrm{O}_{7}: \mathrm{C}, 46.92 ; \mathrm{H}, 6.19 ; \mathrm{N}, 15.63 ; \mathrm{O}$, 31.25. Found: C, 46.88; H, 6.23; N, 15.67; O, 31.27\%.

\section{Boc-gAsn-COOSu (3s)}

White solid, IR: $1715,1750,1785 \mathrm{~cm}^{-1} ;{ }^{1} \mathrm{H}$ NMR (DMSO): $\delta 1.36(9 \mathrm{H}$, s), $3.0(6 \mathrm{H}, \mathrm{m}), 4.8(1 \mathrm{H}, \mathrm{s}), 5.0(2 \mathrm{H}, \mathrm{s}), 5.7(\mathrm{~m}, 1 \mathrm{H}) ;{ }^{13} \mathrm{C} \mathrm{NMR}$ (DMSO): $\delta$ 25.4, 28.0, 42.2, 62.0, 81.0, 149.9, 155.9, 172.3, 174.4. Anal. calcd. for $\mathrm{C}_{13} \mathrm{H}_{20} \mathrm{~N}_{4} \mathrm{O}_{7}$ : C, 45.35; H, 5.85; N, 16.27; O, 32.53. Found: C, 45.30; $\mathrm{H}, 5.90 ; \mathrm{N}, 16.30 ; \mathrm{O}, 32.56 \%$.

\section{Boc-gHis(Z)-COOSu (3t)}

White solid, IR: $1710,1745,1785 \mathrm{~cm}^{-1} ;{ }^{1} \mathrm{H}$ NMR (DMSO): $\delta 1.37(9 \mathrm{H}$, s), $3.1(6 \mathrm{H}, \mathrm{m}), 5.2(2 \mathrm{H}, \mathrm{s}), 7.0(6 \mathrm{H}, \mathrm{m}), 7.6(\mathrm{~m}, 1 \mathrm{H}) ;{ }^{13} \mathrm{C}$ NMR (DMSO): $\delta 25.4,28.1,31.4,63.5,64.6,81.0,107.8,122.2,127.88,127.95,128.4$, 128.9, 138.1, 146.1, 152.0, 153.8, 160.1, 172.3. Anal. calcd. for $\mathrm{C}_{23} \mathrm{H}_{27} \mathrm{~N}_{5} \mathrm{O}_{8}$ : C, 55.09; H, 5.43; N, 13.97; O, 25.52. Found: C, 55.02; $\mathrm{H}, 5.50 ; \mathrm{N}, 13.90 ; \mathrm{O}, 25.55 \%$. 


\section{General Procedure for the Synthesis of Boc-Dipeptidyl Urea Esters (5a-j)}

To a stirred solution of amino acid methyl ester hydrochloride salt $4(1.3 \mathrm{mmol})$ in THF-DMF $(2: 1,5 \mathrm{~mL})$, NMM $(1.3 \mathrm{mmol})$ and $O$ succinimidyl methylcarbamate $3(1 \mathrm{mmol})$ were successively added and stirred at $\mathrm{rt}$ until the completion of the reaction. After the completion of the reaction, the solvent was evaporated under reduced pressure, and the residue was triturated with water, filtered, and finally recrystallized with DMSO-water to afford the pure urea esters $\mathbf{5 a - j}$ as crystalline off-white solids.

\section{Data}

Boc-Val- $\psi(\mathrm{NH}-\mathrm{CO}-\mathrm{NH})-\mathrm{Ala}-\mathrm{OMe}$ (5a)

Yield: 77\%; mp $170{ }^{\circ} \mathrm{C} ;{ }^{1} \mathrm{H}$ NMR (DMSO): $\delta 0.93(6 \mathrm{H}, \mathrm{t}), 1.15(3 \mathrm{H}, \mathrm{d})$, $1.35(9 \mathrm{H}, \mathrm{s}), 1.85(1 \mathrm{H}, \mathrm{m}), 3.6(3 \mathrm{H}, \mathrm{s}), 3.8-3.95(2 \mathrm{H}, \mathrm{m}), 5.15(1 \mathrm{H}, \mathrm{m}), 6.4$ $(1 \mathrm{H}, \mathrm{d}), 6.5(1 \mathrm{H}, \mathrm{d}) ;{ }^{13} \mathrm{C}$ NMR (DMSO): $\delta 18.5,18.8,28.6,32.9,48.3$, 52.0, 60.6, 63.0, 78.0, 155.0, 156.6, 174.5; ESI MS: m/z 319.2 [M+H] $]^{+}$. Anal. calcd. for $\mathrm{C}_{14} \mathrm{H}_{27} \mathrm{~N}_{3} \mathrm{O}_{5}: \mathrm{C}, 52.98 ; \mathrm{H}, 8.57 ; \mathrm{N}, 13.24$. Found: $\mathrm{C}$, $53.20 ; \mathrm{H}, 8.52 ; \mathrm{N}, 13.38 \%$.

Boc-Phe- $\psi(\mathrm{NH}-\mathrm{CO}-\mathrm{NH})-\mathrm{Val}-\mathrm{OMe}$ (5b)

Yield: $72 \%$; mp $140{ }^{\circ} \mathrm{C} ;{ }^{1} \mathrm{H}$ NMR (DMSO): $\delta 0.93(6 \mathrm{H}, \mathrm{t}), 1.3(9 \mathrm{H}, \mathrm{s}), 1.8$ $(1 \mathrm{H}, \mathrm{m}), 2.85(2 \mathrm{H}, \mathrm{d}), 3.6(3 \mathrm{H}, \mathrm{s}), 3.8-3.95(2 \mathrm{H}, \mathrm{m}), 5.2(1 \mathrm{H}, \mathrm{m}), 6.35(1 \mathrm{H}$, br), 6.45 (1H, d), 7.15-7.3 (5H, m); ${ }^{13}$ C NMR (DMSO): $\delta 18.7,19.5,28.3$, 29.5, 37.1, 54.7, 57.5, 63.1, 78.2, 126.9, 127.6, 129.1, 137.9, 155.3, 156.9, 174.5; ESI MS: $m / z 406.7[\mathrm{M}+\mathrm{H}]^{+}$. Anal. calcd. for $\mathrm{C}_{21} \mathrm{H}_{31} \mathrm{~N}_{3} \mathrm{O}_{5}$ : C, $62.20 ; \mathrm{H}, 7.71 ; \mathrm{N}, 10.36$. Found: $\mathrm{C}, 61.90 ; \mathrm{H}, 7.52 ; \mathrm{N}, 10.38 \%$.

\section{Boc-Gly- $\psi(\mathrm{NH}-\mathrm{CO}-\mathrm{NH})-\mathrm{Phe}-\mathrm{OMe}(\mathbf{5 c})$}

Yield: $74 \%$; mp 120-22 ${ }^{\circ}$; ${ }^{1} \mathrm{H}$ NMR (DMSO): $\delta 1.35(9 \mathrm{H}, \mathrm{s}), 2.85(2 \mathrm{H}$, d), $3.6(3 \mathrm{H}, \mathrm{s}), 3.85(3 \mathrm{H}, \mathrm{m}), 5.2(1 \mathrm{H}, \mathrm{m}), 6.3(1 \mathrm{H}, \mathrm{br}), 6.5(1 \mathrm{H}, \mathrm{d}), 7.2-7.4$ (5H, m); ${ }^{13} \mathrm{C}$ NMR (DMSO): $\delta 28.7,37.2,53.9,63.0,64.7,78.1,126.9$, 127.5, 129.1, 137.7, 155.3, 156.7, 173.8; ESI MS: $m / z 353.2[\mathrm{M}+\mathrm{H}]^{+}$. Anal. calcd. for $\mathrm{C}_{17} \mathrm{H}_{25} \mathrm{~N}_{3} \mathrm{O}_{5}: \mathrm{C}, 58.11 ; \mathrm{H}, 7.17 ; \mathrm{N}, 11.96$. Found: $\mathrm{C}$, $58.38 ; \mathrm{H}, 7.52 ; \mathrm{N}, 11.78 \%$. 


\section{Boc-Phe- $\psi(\mathrm{NH}-\mathrm{CO}-\mathrm{NH})-\mathrm{Ala}-\mathrm{OMe}$ (5d)}

Yield: $76 \%$; mp $138-139^{\circ} \mathrm{C} ;{ }^{1} \mathrm{H}$ NMR (DMSO): $\delta 1.15(3 \mathrm{H}, \mathrm{d}), 1.35(9 \mathrm{H}$, s), $2.84(2 \mathrm{H}, \mathrm{d}), 3.65(3 \mathrm{H}, \mathrm{s}), 3.85(2 \mathrm{H}, \mathrm{m}), 5.15(1 \mathrm{H}, \mathrm{d}), 6.35(1 \mathrm{H}, \mathrm{d}), 6.5$ $(1 \mathrm{H}, \mathrm{d}), 7.15-7.4(5 \mathrm{H}, \mathrm{m}) ;{ }^{13} \mathrm{C}$ NMR (DMSO): $\delta 17.1,28.7,37.1,48.7$, 54.7, 63.5, 78.3, 126.7, 127.9, 129.2, 137.9, 154.8, 156.3, 174.5; ESI MS: $m / z$ 366.1 $[\mathrm{M}+\mathrm{H}]^{+}$. Anal. calcd. for $\mathrm{C}_{18} \mathrm{H}_{27} \mathrm{~N}_{3} \mathrm{O}_{5}: \mathrm{C}, 59.16 ; \mathrm{H}, 7.45$; $\mathrm{N}, 11.50$. Found: C, 59.30; H, 7.52; N, 11.20\%.

\section{Boc-Ser(Bzl)- $\psi(\mathrm{NH}-\mathrm{CO}-\mathrm{NH})-$ Gly-OMe (5e)}

Yield: $78 \%$; mp $124-126^{\circ} \mathrm{C} ;{ }^{1} \mathrm{H}$ NMR (DMSO): $\delta 1.35(9 \mathrm{H}, \mathrm{s}), 3.6(3 \mathrm{H}$, s), 3.7-3.85 (5H, m), $4.48(2 \mathrm{H}, \mathrm{s}), 5.25(1 \mathrm{H}, \mathrm{br}), 6.3(1 \mathrm{H}, \mathrm{d}), 6.45(1 \mathrm{H}, \mathrm{d})$, 7.3-7.4 (5H, m); ${ }^{13} \mathrm{C}$ NMR (DMSO): $\delta 28.5,51.7,57.7,63.7,65.0,71.9$, 78.0, 126.6, 129.3, 130.5, 138.0, 155.3, 156.8, 174.5; ESI MS: $m / z 383.1$ $[\mathrm{M}+\mathrm{H}]^{+}$. Anal. calcd. for $\mathrm{C}_{18} \mathrm{H}_{27} \mathrm{~N}_{3} \mathrm{O}_{6}: \mathrm{C}, 56.68 ; \mathrm{H}, 7.15 ; \mathrm{N}, 11.02$. Found: C, 56.38; H, 7.20; N, $11.10 \%$.

\section{Boc-Asp(OBzl)- $\psi(\mathrm{NH}-\mathrm{CO}-\mathrm{NH})-\mathrm{Gly}-\mathrm{OMe}$ (5f)}

Yield: $70 \%$; mp $120-121^{\circ} \mathrm{C} ;{ }^{1} \mathrm{H}$ NMR (DMSO): $\delta 1.4(9 \mathrm{H}, \mathrm{s}), 2.55(2 \mathrm{H}$, d), $3.6(3 \mathrm{H}, \mathrm{s}), 3.85(3 \mathrm{H}, \mathrm{m}), 5.0(1 \mathrm{H}, \mathrm{m}), 5.1(2 \mathrm{H}, \mathrm{s}), 6.35(1 \mathrm{H}, \mathrm{d}), 6.5$ $(1 \mathrm{H}, \mathrm{t}), 7.25-7.35(5 \mathrm{H}, \mathrm{m}) ;{ }^{13} \mathrm{C}$ NMR (DMSO): $\delta 28.1,38.0,49.5,61.5$, 62.7, 64.7, 78.5, 127.6, 128.5, 129.3, 136.7, 155.0, 156.8, 174.5; ESI MS: $m / z$ 425.1 $[\mathrm{M}+\mathrm{H}]^{+}$. Anal. calcd. for $\mathrm{C}_{20} \mathrm{H}_{29} \mathrm{~N}_{3} \mathrm{O}_{7}: \mathrm{C}, 56.73 ; \mathrm{H}, 6.90$; N, 9.92. Found: C, 56.80; H, 6.52; N, 10.38\%.

\section{Boc-Glu(OBzl)- $\psi(\mathrm{NH}-\mathrm{CO}-\mathrm{NH})-\mathrm{Leu}-\mathrm{OMe} \mathbf{( 5 g})$}

Yield: $74 \%$; mp $139-140{ }^{\circ} \mathrm{C} ;{ }^{1} \mathrm{H}$ NMR (DMSO): $\delta 0.91$ (6H, d), $1.3-1.45$ $(11 \mathrm{H}, \mathrm{s}), 1.65(1 \mathrm{H}, \mathrm{m}), 2.55(2 \mathrm{H}, \mathrm{m}), 2.9(2 \mathrm{H}, \mathrm{m}), 3.65(3 \mathrm{H}, \mathrm{s}), 3.8-3.9$ $(2 \mathrm{H}, \mathrm{m}), 5.15(2 \mathrm{H}, \mathrm{s}), 5.3(1 \mathrm{H}, \mathrm{d}), 6.35(1 \mathrm{H}, \mathrm{d}), 6.5(1 \mathrm{H}, \mathrm{d}), 7.3-7.4(5 \mathrm{H}$, $\mathrm{m}) ;{ }^{13} \mathrm{C}$ NMR (DMSO): $\delta 22.1,23.1,24.7,28.6,37.9,39.7,41.9,50.9$, 51.7, 61.9, 63.1, 78.7, 126.7, 127.6, 128.9, 137.7, 155.3, 156.8, 157.5, 178.1; ESI MS: $m / z 480.2[\mathrm{M}+\mathrm{H}]^{+}$. Anal. calcd. for $\mathrm{C}_{24} \mathrm{H}_{37} \mathrm{~N}_{3} \mathrm{O}_{7}: \mathrm{C}$, 60.11; H, 7.78; N, 8.76. Found: C, 59.80; H, 7.52; N, 9.10\%.

\section{Boc-Ile- $\psi(\mathrm{NH}-\mathrm{CO}-\mathrm{NH})-\mathrm{Phe}-\mathrm{OMe}$ (5h)}

Yield: $72 \%$; mp $135-137^{\circ} \mathrm{C} ;{ }^{1} \mathrm{H}$ NMR (DMSO): $\delta 0.91(6 \mathrm{H}, \mathrm{m}), 1.13(1 \mathrm{H}, \mathrm{m})$, $1.35(9 \mathrm{H}, \mathrm{s}), 1.55(2 \mathrm{H}, \mathrm{m}), 2.85(2 \mathrm{H}, \mathrm{d}), 3.65(3 \mathrm{H}, \mathrm{s}), 3.85(2 \mathrm{H}, \mathrm{m}), 5.15(1 \mathrm{H}, \mathrm{d})$, 
$6.35(1 \mathrm{H}, \mathrm{d}), 6.5(1 \mathrm{H}, \mathrm{d}), 7.3-7.45(5 \mathrm{H}, \mathrm{m}) ;{ }^{13} \mathrm{C}$ NMR (DMSO): $\delta 11.7$, 15.5, 25.5, 28.3, 37.5, 55.1, 58.7, 63.5, 78.3, 126.7, 127.9, 129.2, 137.9, 154.8, 156.3, 174.5; ESI MS: $\mathrm{m} / \mathrm{z} 409.4[\mathrm{M}+\mathrm{H}]^{+}$. Anal. calcd. for $\mathrm{C}_{21} \mathrm{H}_{33} \mathrm{~N}_{3} \mathrm{O}_{5}: \mathrm{C}, 61.90 ; \mathrm{H}, 8.16 ; \mathrm{N}, 10.31$. Found: $\mathrm{C}, 62.20 ; \mathrm{H}, 8.52 ; \mathrm{N}, 10.38 \%$.

Boc-Tyr- $\psi(\mathrm{NH}-\mathrm{CO}-\mathrm{NH})-$ Ala-OMe (5i)

Yield: $70 \%$; mp $140-42^{\circ} \mathrm{C} ;{ }^{1} \mathrm{H}$ NMR (DMSO): $\delta 1.2(3 \mathrm{H}, \mathrm{s}), 1.37(9 \mathrm{H}, \mathrm{s})$, $3.1(2 \mathrm{H}, \mathrm{m}), 3.71(3 \mathrm{H}, \mathrm{s}), 3.8(\mathrm{~m}, 1 \mathrm{H}), 6.2(\mathrm{~m}, 2 \mathrm{H}), 7.0(\mathrm{~m}, 4 \mathrm{H}) ;{ }^{13} \mathrm{C} \mathrm{NMR}$ (DMSO): $\delta 16.1,28.0,40.3,50.6,52.1,66.9,81.0,119.1,127.1,128.7$, 128.4, 1548, 158.4, 159.6, 172.1; ESI MS: $\mathrm{m} / \mathrm{z} 382.4[\mathrm{M}+\mathrm{H}]^{+}$. Anal. calcd. for $\mathrm{C}_{18} \mathrm{H}_{27} \mathrm{~N}_{3} \mathrm{O}_{8}$ : C, 56.68; H, 7.13; N, 11.02; O, 25.17. Found: C, 56.62; H, 7.18; N, 11.06, O, 25.20\%.

Boc-Trp(Z)- $\psi(\mathrm{NH}-\mathrm{CO}-\mathrm{NH})-$ Val-OMe (5j)

Yield: 69\%; mp 148-50 ${ }^{\circ} \mathrm{C} ;{ }^{1} \mathrm{H}$ NMR (DMSO): $\delta 0.9(6 \mathrm{H}, \mathrm{s}), 1.36(9 \mathrm{H}, \mathrm{s})$, $1.8(\mathrm{~m}, 1 \mathrm{H}), 3.4(\mathrm{~m}, 2 \mathrm{H}), 3.7(3 \mathrm{H}, \mathrm{s}), 4.4(1 \mathrm{H}, \mathrm{m}), 5.2(2 \mathrm{H}, \mathrm{s}), 5.8(\mathrm{~m}, 1 \mathrm{H})$, 7.0-7.9 (10H, m); ${ }^{13} \mathrm{C}$ NMR (DMSO): $\delta 18.1,28.0,34.2,34.3,57.2,29.8$, 63.4, 67.7, 79.3, 108.8, 119.2, 122.2, 122.5, 123.7, 124.8, 127.6, 127.9, $128.5,136.0,145.4,146.8,159.8,161.0,173.1$; ESI MS: $\mathrm{m} / \mathrm{z} 567.3$ $[\mathrm{M}+\mathrm{H}]^{+}$. Anal. calcd. for $\mathrm{C}_{30} \mathrm{H}_{38} \mathrm{~N}_{4} \mathrm{O}_{7}: \mathrm{C}, 63.59 ; \mathrm{H}, 6.76 ; \mathrm{N}, 9.89 ; \mathrm{O}$, 19.76. Found: C, 63.55; H, 6.80; N, 9.91; O, 19.80\%.

\section{Boc-Dipeptidyl Urea Acids (8a-d): General Procedure}

To a stirred suspension of amino acid $6(1 \mathrm{mmol})$ in $\mathrm{CH}_{2} \mathrm{Cl}_{2}(5 \mathrm{~mL})$ freshly distilled TMS-Cl $(2.2 \mathrm{mmol})$ and TEA $(2.2 \mathrm{mmol})$ were added and refluxed for $2 \mathrm{~h}$. The reaction mixture was cooled to rt, $O$-succinimidyl methylcarbamate $3(1 \mathrm{mmol})$ in THF-DMF $(2: 1,5 \mathrm{~mL})$ solution was added, and it was stirred for $30 \mathrm{~min}$ at rt. After the completion of the reaction, the solvent was evaporated under reduced pressure, and the residue was triturated with water (to remove side product formed during the reaction), filtered, and recrystallized using DMSO-water to afford the

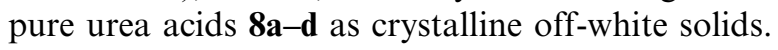

\section{Data}

Boc-Leu- $\psi(\mathrm{NH}-\mathrm{CO}-\mathrm{NH})-\mathrm{Ala}-\mathrm{OH}$ (8a)

Yield: $80 \%$; mp $140-141^{\circ} \mathrm{C} ;{ }^{1} \mathrm{H}$ NMR (DMSO): $\delta 0.93(6 \mathrm{H}, \mathrm{d}), 1.15(3 \mathrm{H}$, d), $1.3(9 \mathrm{H}, \mathrm{s}), 1.35(2 \mathrm{H}, \mathrm{t}), 1.6(1 \mathrm{H}, \mathrm{m}), 3.75-3.8(2 \mathrm{H}, \mathrm{m}), 5.2(1 \mathrm{H}, \mathrm{br})$, 
6.3-6.45 (2H, m), $8.4(1 \mathrm{H}, \mathrm{d}) ;{ }^{13} \mathrm{C}$ NMR (DMSO): $\delta 17.4,22.1,23.3,24.7$, 28.5, 40.1, 49.2, 51.3, 78.1, 155.2, 156.9, 177.8; $\mathrm{MS}\left(\mathrm{FAB}^{+}\right): \mathrm{m} / z$ 318.1. Anal. calcd. for $\mathrm{C}_{14} \mathrm{H}_{27} \mathrm{~N}_{3} \mathrm{O}_{5}: \mathrm{C}, 52.98 ; \mathrm{H}, 8.57 ; \mathrm{N}, 13.24$. Found: $\mathrm{C}$, $52.66 ; \mathrm{H}, 8.57$; N, $13.20 \%$.

\section{Boc-Phe- $\psi(\mathrm{NH}-\mathrm{CO}-\mathrm{NH})-\mathrm{Ala}-\mathrm{OH}(\mathbf{8 b})$}

Yield: $78 \%$; mp $137-138^{\circ} \mathrm{C} ;{ }^{1} \mathrm{H}$ NMR (DMSO): $\delta 1.16(3 \mathrm{H}, \mathrm{d}), 1.31(9 \mathrm{H}$, s), $2.85(2 \mathrm{H}, \mathrm{d}), 3.8-3.9(2 \mathrm{H}, \mathrm{m}), 5.3(1 \mathrm{H}, \mathrm{br}), 6.4-6.4(2 \mathrm{H}, \mathrm{m}), 7.15-7.3$ $(5 \mathrm{H}, \mathrm{m}), 8.35(1 \mathrm{H}, \mathrm{d}) ;{ }^{13} \mathrm{C}$ NMR (DMSO): $817.3,28.1,37.5,48.7,54.1$, 77.2, 126.7, 129.1, 130.2, 137.9, 155.1, 156.7, 177.5; $\mathrm{MS}\left(\mathrm{FAB}^{+}\right): m / z$ 352.2. Anal. calcd. for $\mathrm{C}_{17} \mathrm{H}_{25} \mathrm{~N}_{3} \mathrm{O}_{5}: \mathrm{C}, 58.11 ; \mathrm{H}, 7.17 ; \mathrm{N}, 11.96$. Found: C, 58.16; H, 7.40; N, $11.70 \%$.

\section{Boc-Leu- $\psi(\mathrm{NH}-\mathrm{CO}-\mathrm{NH})-\mathrm{Gly}-\mathrm{OH}$ (8c)}

Yield: $75 \%$; mp $123-125^{\circ} \mathrm{C} ;{ }^{1} \mathrm{H}$ NMR (DMSO): $\delta 0.93(6 \mathrm{H}, \mathrm{d}), 1.3(9 \mathrm{H}$, s), $1.4(2 \mathrm{H}, \mathrm{m}), 1.65(1 \mathrm{H}, \mathrm{m}), 3.5(2 \mathrm{H}, \mathrm{d}), 3.7(1 \mathrm{H}, \mathrm{m}), 5.2(1 \mathrm{H}, \mathrm{d}), 6.1$ $(1 \mathrm{H}, \mathrm{br}), 6.35(1 \mathrm{H}, \mathrm{d}), 8.35(1 \mathrm{H}, \mathrm{d}) ;{ }^{13} \mathrm{C}$ NMR (DMSO): $\delta 22.0,23.1$, 24.7, 28.1, 40.5, 41.9, 52.0, 75.9, 155.2, 156.9, 178.8; $\mathrm{MS}\left(\mathrm{FAB}^{+}\right): m / z$ 304.2. Anal. calcd. for $\mathrm{C}_{13} \mathrm{H}_{25} \mathrm{~N}_{3} \mathrm{O}_{5}: \mathrm{C}, 51.47 ; \mathrm{H}, 8.31 ; \mathrm{N}, 13.85$. Found: C, $51.20 ; \mathrm{H}, 8.52 ; \mathrm{N}, 13.78 \%$.

\section{Boc-Val- $\psi(\mathrm{NH}-\mathrm{CO}-\mathrm{NH})-\mathrm{Phe}-\mathrm{OH}$ (8d)}

Yield: $72 \%$; mp $128-130^{\circ} \mathrm{C} ;{ }^{1} \mathrm{H}$ NMR (DMSO): $\delta 0.80-0.91(6 \mathrm{H}, \mathrm{t}$ ), 1.35 $(9 \mathrm{H}, \mathrm{s}), 1.85(1 \mathrm{H}, \mathrm{m}), 2.8(2 \mathrm{H}, \mathrm{d}), 4.3(1 \mathrm{H}, \mathrm{m}), 4.8(1 \mathrm{H}, \mathrm{m}), 5.5(1 \mathrm{H}, \mathrm{d})$, 6.1-6.3 (2H, br), 7.13-7.25 (5H, m), $8.35(1 \mathrm{H}, \mathrm{d}) ;{ }^{13} \mathrm{C}$ NMR (DMSO): $\delta$ $18.5,19.3,26.1,29.2,37.2,54.9,62.1,78.1,126.7,128.5,129.2,137.5$, 154.5, 156.7, 176.2; $\mathrm{MS}\left(\mathrm{FAB}^{+}\right): \mathrm{m} / z$ 380.0. Anal. calcd. for $\mathrm{C}_{19} \mathrm{H}_{29} \mathrm{~N}_{3} \mathrm{O}_{5}: \mathrm{C}, 60.14 ; \mathrm{H}, 7.70 ; \mathrm{N}, 11.07$. Found: $\mathrm{C}, 60.45 ; \mathrm{H}, 7.57 ; \mathrm{N}$, $11.20 \%$.

\section{Test for Racemization of Methylcarbamate $3 \mathrm{f}$}

To a stirred solution of methylcarbamate $3 \mathbf{f}(0.377 \mathrm{~g}, 1 \mathrm{mmol})$ in THFDMF $(5 \mathrm{~mL})$, optically pure $(\mathrm{R})-(+)-1$-phenylethylamine $(0.121 \mathrm{~g}$, $1 \mathrm{mmol}$ ) was added, and the mixture was stirred at $25^{\circ} \mathrm{C}$ for $30 \mathrm{~min}$. After routine workup, it resulted in the diastereomer 10a. The solid was recrystallized using DMSO-water. Mp $165^{\circ} \mathrm{C} ;[\alpha]_{\mathrm{D}}{ }^{25}:+24.8$ (c 1, DMSO); ${ }^{1} \mathrm{H}$ NMR (DMSO): $\delta$ 1.2-1.4 (12H, t), $2.9(2 \mathrm{H}, \mathrm{d}), 3.3(1 \mathrm{H}, \mathrm{m}), 4.7(1 \mathrm{H}, \mathrm{m})$, 
$5.1(1 \mathrm{H}$, br $), 6.2(1 \mathrm{H}, \mathrm{s}), 6.55(1 \mathrm{H}, \mathrm{m}), 7.2-7.35(10 \mathrm{H}, \mathrm{m}) ; \mathrm{MS}$ $\left(\mathrm{FAB}^{+}\right): m / z$ 384.1. When this experiment was repeated using $3 \mathbf{f}$ with optically pure $(\mathrm{S})-(-)-1$-phenylethylamine $(0.121 \mathrm{~g}, 1 \mathrm{mmol})$, the other diastereomer $10 \mathrm{~b}$ was isolated. $\mathrm{Mp} 160-161^{\circ} \mathrm{C} ;[\alpha]_{\mathrm{D}}^{25}:-25.6$ (c 1, DMSO); ${ }^{1} \mathrm{H}$ NMR (DMSO): $\delta$ 1.2-1.4 (12H, t), $2.9(2 \mathrm{H}, \mathrm{d}), 3.3(1 \mathrm{H}$, $\mathrm{m}), 4.7(1 \mathrm{H}, \mathrm{m}), 5.1(1 \mathrm{H}, \mathrm{br}), 6.2(1 \mathrm{H}, \mathrm{s}), 6.55(1 \mathrm{H}, \mathrm{m}), 7.2-7.35(10 \mathrm{H}$, m); $\mathrm{MS}\left(\mathrm{FAB}^{+}\right): \mathrm{m} / z$ 384.1. Anal. calcd. for $\mathrm{C}_{22} \mathrm{H}_{29} \mathrm{~N}_{3} \mathrm{O}_{3}: \mathrm{C}, 68.90 ; \mathrm{H}$, 7.62; N, 10.96. Found: C, 68.91; H, 7.23; N, 10.59\%. The experiment was repeated using $\mathbf{3 f}$ with racemic 1-phenylethylamine $(0.121 \mathrm{~g}, 1 \mathrm{mmol})$. The corresponding diastereomer 10c was obtained and isolated. Mp 155$157^{\circ} \mathrm{C} ;{ }^{1} \mathrm{H}$ NMR (DMSO): $\delta$ 1.2-1.4 (12H, dd), $2.9(2 \mathrm{H}, \mathrm{d}), 3.3(1 \mathrm{H}, \mathrm{m})$, $4.7(1 \mathrm{H}, \mathrm{m}), 5.1(1 \mathrm{H}, \mathrm{br}), 6.2(1 \mathrm{H}, \mathrm{s}), 6.55(1 \mathrm{H}, \mathrm{m}), 7.2-7.35(10 \mathrm{H}, \mathrm{m}) ; \mathrm{MS}$ $\left(\mathrm{FAB}^{+}\right): m / z$ 384.0. Anal. calcd. for $\mathrm{C}_{22} \mathrm{H}_{29} \mathrm{~N}_{3} \mathrm{O}_{3}: \mathrm{C}, 68.90 ; \mathrm{H}, 7.62 ; \mathrm{N}$, 10.96. Found: C, 68.81; H, 7.29; N, 10.60\%.

\section{ACKNOWLEDGMENTS}

Authors thank the Department of Science and Technology, Government of India, for financial assistance. Authors also thank Sophisticated Instruments Facility, Indian Institute of Science (IISc), for NMR data and P. Balaram, Molecular Biophysics Unit (MBU), IISc, Bangalore, for mass spectral data.

\section{REFERENCES}

1. Zhang, X.; Rodrigues, J.; Evans, L.; Hinkle, B.; Ballantyne, L.; Pena, M. Formation of urea dipeptides from carbonyldiimidazole: Application toward the protease inhibitors GE 20372 and MAPI. J. Org. Chem. 1997, 62, 64206423.

2. Tamilarasu, N.; Huq, I.; Rana, T. M. Targeting RNA with peptidomimetic oligomers in human cells. Bioorg. Med. Chem. Lett. 2001, 11, 505-507.

3. Holmes, D. L.; Smith, E. M.; Nowick, J. S. Solid-phase synthesis of artificial ?-sheets. J. Am. Chem. Soc. 1997, 119, 7665-7669.

4. Nowick, J. S.; Pairish, M.; Lee, I. Q.; Holmes, D. L.; Ziller, J. W. An extended ?-strand mimic for a larger artificial ?-sheet. J. Am. Chem. Soc. 1997, 119, 5413-5424

5. Fischer, V.; Semetey, V.; Lozano, J-M.; Schaffner, A-P.; Briand, J-P.; Didierjean, C.; Guichard, G. Succinimidyl carbamate derivatives from N-protected ?amino acids and dipeptides- synthesis of ureidopeptides and oligourea/peptide eptide hybrids. Eur. J. Chem. 2007, 2511-2525, and references therein.

6. Okada, Y.; Tsuda, Y.; Yagyu, M. Amino acids and peptides. VI. Curtius rearrangement of acyl amino acid and peptide azides and reactivity of the isocyanates. Chem. Pharm. Bull. 1980, 28, 2254. 
7. Guichard, G.; Semetey, V.; Didierjean, C.; Aubry, A.; Briand, J.-P.; Rodriguez, M. Effective preparation of O-succinimidyl-2-(tert-butoxycarbonylamino)ethylcarbamate derivatives from ?-amino acids. Application to the synthesis of urea-containing pseudopeptides and oligoureas. J. Org. Chem. 1999, 64, 8702.

8. Guichaard, G.; Semetey, V.; Rodriguez, M.; Briand, J.-P. Solid phase synthesis of oligoureas using O-succinimidyl-(9H-fluoren-9-ylmethoxycarbonylamino)ethylcarbamate derivatives as activated monomers. Tetrahedron Lett. 2000, 41, 1553.

9. Semetey, V.; Hemmerlin, C.; Didierjean, C.; Schaffner, A.-P.; Giner, A. G.; Aubry, A.; Briand, J.-P.; Marraud, M.; Guichard, G. Unexpected stability of the urea cistrans isomer in urea-containing model pseudopeptides. Org. Lett. 2001, 3, 3843.

10. Semetey, V.; Schaffner, A.-P.; Marraud, M.; Didierjean, C.; Aubry, A.; Rodriguez, M.; Briand, J.-P.; Guichard, G. In Peptides; J. Martinez, J.-A. Fehrentz (Eds.), The 26th European Peptide Symposium, Montpellier, EDK, Paris, 2000, pp. 161-162. 